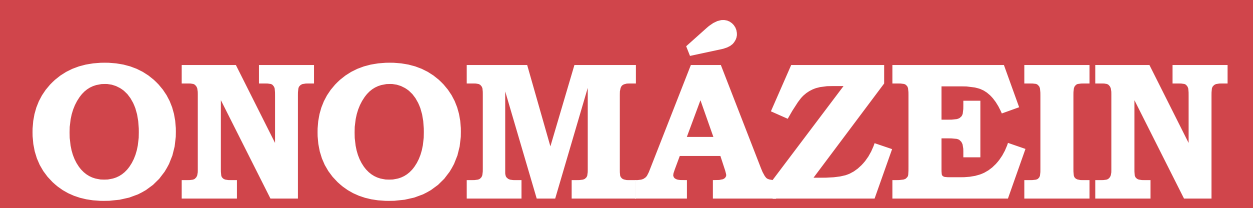

Revista semestral de lingüística, filología y traducción
PONTIFICIA UNIVERSIDAD CATÓLICA DE CHILE FACULTAD DE LETRAS

\title{
La metáfora gramatical en los textos escolares de Ciencias Sociales en español ${ }^{1}$
}

Grammatical metaphor in Social Sciences textbooks in Spanish

\section{Gillian Moss}

Universidad del Norte

Colombia

\section{Diana Chamorro Miranda}

Universidad del Norte

Colombia
Norma Barletta Manjarrés

Universidad del Norte

Colombia

Jorge Mizuno Haydar

Universidad del Norte

Colombia

Gillian Moss: Departamento de Lenguas, Universidad del Norte. Colombia Correo electrónico: mgilmoss@yahoo.com.ar

Norma Barletta: Departamento de Lenguas, Universidad del Norte. Colombia Correo electrónico: nbarlett@uninorte.edu.co

Diana Chamorro: Departamento de Lenguas, Universidad del Norte. Colombia Correo electrónico: dchamorro@uninorte.edu.co

Jorge Mizuno Haydar: Departamento de Lenguas, Universidad del Norte. Colombia Correo electrónico: jmizzuno@uninorte.edu.co 
Comprender algo equivale a transformarlo en sentido; y el resultado de esta transformación es lo que llamamos "conocimiento"; [...] la comprensión y el conocimiento son procesos semióticos [...] y la "central eléctrica" de estos procesos es la gramática (Halliday, 2004b, traducido por Moss).

\section{Resumen}

En el presente artículo abordamos el estudio de la metáfora gramatical en español, específicamente en textos escolares de Ciencias Sociales empleados en Colombia. Nuestro interés se centra en analizar, desde la perspectiva de la Gramática Sistémico-Funcional (Halliday, 1994, 2004b), las particularidades de la metáfora gramatical ideacional en español y sus implicaciones para la formación ciudadana y el aprendizaje. El corpus lo conforman tres capítulos de textos para octavo grado que aluden a la Revolución Industrial. El proceso seguido para realizar nuestros análisis nos ha permitido identificar la presencia de metáforas gramaticales en los términos hallidayanos: nominalizaciones de procesos y cualidades, y el uso de verbos causales en lugar de conjunciones. Asimismo, hemos identificado otros tipos de metáforas: cláusulas no ergativas, presente histórico, participantes no animados. En el artículo resaltamos la importancia del desempaque de las metáforas en los procesos de análisis y también de enseñanza y aprendizaje, ya que a través de él se visibiliza la información escondida en la metáfora.

Palabras clave: metáfora gramatical; nominalización; ideología; aprendizaje.

\section{Abstract}

This paper addresses the study of grammatical metaphor in Spanish, specifically in Social science textbooks in use in Colombian secondary schools. Our main interest is to analyze, from a Systemic Functional Grammar perspective (Halliday, 1994, 2004b), the particular characteristics of ideational grammatical metaphors in Spanish and their implications for citizenship education and for learning processes. The corpus is made up of chapters about the Industrial Revolution from three eighth-grade texts. Our analysis has identified the presence of grammatical metaphors in Hallidayan terms: nominalizations of processes and qualities and the use of causal verbs in place of conjunctions. We have also identified other types of metaphor: nonergative clauses, historical present tense, and inanimate participants. The paper also stresses the importance of unpacking metaphors during the process of analysis and also during teaching and learning processes, since unpacking sheds light on information hidden in the metaphor.

Keywords: grammatical metaphor; nominalization; ideology; learning.

1 Este artículo es producto de la investigación "Comparación de los rasgos discursivos de dos textos escolares de ciencias sociales (1999, 2010)", adelantada por el Colectivo Urdimbre y auspiciada por la Dirección de Investigación, Desarrollo e Innovación (DIDI), Universidad del Norte, Barranquilla, Colombia. 


\section{Introducción}

La ciencia, en su devenir histórico, ha ido construyendo un discurso propio diferente del discurso de la cotidianidad. Este discurso de la ciencia nos presenta la interpretación de la realidad desde la perspectiva de las diversas ramas del saber, con estructuras lexicogramaticales características del lenguaje escrito. En esa evolución del lenguaje científico, la metáfora gramatical (Halliday, 1994, 2004a, 2004b) se ha constituido en un recurso importante que ayuda a representar sintéticamente los eventos del mundo tanto natural como social. En este artículo trataremos sobre este tema, mirado desde la perspectiva de su utilización en los manuales escolares y los posibles problemas que puede presentar para los aprendices. Queremos mostrar brevemente la propuesta de Halliday y complementarla con los resultados que hemos obtenido en nuestras investigaciones sobre el discurso de los textos escolares y el aprendizaje. En la primera parte del artículo esbozaremos la teoría de Halliday sobre la evolución del lenguaje; luego expondremos la conceptualización de metáfora gramatical desde la Lingüística Sistémico-Funcional (metáforas ideacionales e interpersonales) focalizándonos en las metáforas ideacionales, para continuar con los resultados de nuestros análisis, en los que plantearemos otras formas de metáfora gramatical

Por política editorial de la revista, empleamos el masculino como género no marcado, es decir, escribimos en masculino (y no femenino) los sustantivos que, refiriéndose a seres animados, designen a todos los individuos de una especie, tanto femeninos como masculinos, sin distinción de sexos. Los ejemplos usados son extraídos de tres de los diez manuales escolares que nos han servido en nuestras investigaciones de los últimos 20 años: Hipertexto 8 de Editorial Santillana, 2010, Civilización 8 de Editorial Norma, 1994, y Milenio 8 de Editorial Norma, 1997.

\section{La perspectiva hallidayana sobre la metáfora gramatical}

Al tratar sobre los estilos de la prosa, Halliday (1994) propone un continuum: en un extremo, el estilo dinámico, propio del lenguaje oral, al que denomina "estilo dórico", y en el otro, el estilo estático, sinóptico, propio del lenguaje escrito, más formal, al que Ilama "estilo ático". El primer caso, el estilo dórico, se caracteriza porque representa al mundo en términos de ocurrencias, procesos y transformaciones, y porque utiliza una gramática intrincada, con oraciones que normalmente incluyen muchas cláusulas tanto hipotácticas como paratácticas, relacionadas unas con otras. Este tipo de prosa se asocia con el lenguaje oral, pero también puede encontrarse en el lenguaje escrito.

El estilo ático, por su parte, se asocia con la escritura formal y cuidadosamente planificada, pero también puede encontrarse en el lenguaje oral. El mundo, en este caso, es representado como un mundo de cosas en vez de ocurrencias; de productos, en vez de procesos; de ser en vez de transformarse (Halliday, 1994). Con el uso de este tipo de estilo, el autor puede congelar aquello de lo que escribe y lo toma como un todo. Las principales características del estilo ático son la densidad léxica, altos niveles de abstracción (característica del lenguaje de la ciencia), gramática no compleja y las metáforas gramaticales.

En sus estudios del lenguaje de las ciencias, Halliday (2004a) ha identificado una serie de etapas de progresiva abstracción y generalización por las que pasa el aprendiz. La primera etapa sería la del protolenguaje, utilizado únicamente para la comunicación con los seres más allegados. La siguiente etapa está caracterizada por la generalización, representada por el manejo del lenguaje oral de la lengua materna. La etapa posterior se distingue por el nivel de abstracción y corresponde a la lengua escrita. Finalmente, se Ilega al estilo ático caracterizado por altos nive- 
les de abstracción y generalización, expresados por medio de la metáfora gramatical, especialmente la nominalización compleja. Como afirma Vygotsky (1986 [1934]), se parte de la experiencia directa y, en etapas posteriores, el individuo se desprende cada vez más de la experiencia sensible e ingresa al terreno de la conceptualización. Este proceso no se limita exclusivamente a los niños, sino que ocurre durante toda la vida y no necesariamente conduce a la formación del concepto como tal. El pensamiento puede permanecer en estadios anteriores, como, por ejemplo, en lo que Vygotsky denominó "seudoconcepto", en donde la persona tiene una vaga idea de lo que significa un término, sin llegar a comprenderlo completamente.

Este proceso individual (ontogenético) es similar al ocurrido en el desarrollo de las lenguas (filogenético), que en sus inicios probablemente servían para significar las entidades concretas; luego, llegarían a una etapa de generalización, del nombre propio al nombre común, que haría posible la existencia del denominado "sentido común", también llamado "conocimiento cotidiano"; en un nivel mayor de evolución del lenguaje, encontramos la abstracción en la que se pasa de categorizaciones concretas a otras más abstractas, de manera que se re-teoriza el sentido común: son los inicios de la ciencia formal, es decir, el lenguaje escrito. Por último, se llegaría a la etapa de la metáfora gramatical, que se caracteriza por establecer relaciones entre conceptos y producir una nueva re-teorización del conocimiento, estableciendo el conocimiento científico y técnico con sus correspondientes discursos. En otras palabras, es el paso del estilo dórico (lenguaje característico del sentido común, es decir, el lenguaje oral) al estilo ático (lenguaje escrito y más elaborado de la ciencia). A continuación, presentaremos una breve descripción de la teoría hallidayana de la metáfora gramatical.

La metáfora gramatical (Halliday y Martin, 1993; Halliday, 1994, 2004a, 2004b; Halliday y Matthiessen, 1999) es un proceso en donde un componente semántico se construye en la gramática en una forma que no es prototípica; representa uno de los patrones lingüísticos más típicos de una alfabetización avanzada (Colombi, 2000). Una idea o proposición puede ser expresada por medio de diversas estructuras gramaticales, de las cuales una se considera como congruente mientras que las demás son metafóricas; tal es el caso de los procesos, los cuales se expresan de manera congruente mediante verbos y metafóricamente mediante sustantivos. La realización de procesos por medio de sustantivos crea una tensión entre el estrato semántico (el proceso) y el estrato lexicogramatical (el sustantivo) (Halliday, 2004a: xvi). Este fenómeno se considera como metáfora porque crea entidades virtuales que existen solamente en el plano semiótico (Halliday, 2004a: xvii).

La forma congruente de expresar el significado precede a la forma metafórica, tanto en el desarrollo filogenético como en el ontogenético (Halliday, 2004a: xvii). Esto no significa que la realización metafórica sea mejor o peor, o más o menos frecuente. Es una herramienta característica del registro científico ya que permite destilar significados, creando y desarrollando el campo por medio de la definición, clasificación y explicación (Wignell, 1998: 299). Al mismo tiempo, tiene una función discursiva en el desarrollo de una argumentación dinámica y fluida (Halliday, 1998: 202).

La metáfora gramatical puede ser de diversos tipos, los cuales se pueden clasificar en dos grandes grupos, a saber: las ideacionales (Halliday, 2004b: 636), correspondientes a la metafunción ideacional, es decir, aquellos significados que tienen que ver con la construcción de la experiencia: qué ocurre, quién hace qué y a quién, dónde, cuándo, cómo y por qué; y las interpersonales (Halliday, 2004b: 626), correspondientes a la metafunción interpersonal, o sea, los significados que tienen que ver con las relaciones sociales: cómo interactúan las personas y qué sentimientos y puntos de vista intentan compar- 
tir. En este artículo nos concentraremos en las metáforas ideacionales, las cuales, según Martin (1992), pueden ser: nominalizaciones de procesos y cualidades (metáfora experiencial), y el uso de verbos causales en lugar de conjunciones (metáfora lógica)

\subsection{Metáfora ideacional: nominalización}

En este artículo, analizamos las nominalizaciones como entidades léxicas ya existentes y no en términos de los procesos sintácticos que llevan a su formación (Billig, 2008; Fairclough, 2008). Nuestro interés se centra en la escogencia de discursos nominalizados por parte de autores que construyen textos para acercar al estudiante al conocer científico. Este tipo de metáfora gramatical ha sido objeto de varios estudios ya que se considera como uno de los aspectos gramaticales más destacados en el discurso científico y en los libros de texto escolares en las ciencias, tanto en inglés (Eggins y otros, 1993; Halliday y Martin, 1993; Wignell, 1998; Halliday, 1998, 2004a; Martin, 2003) como en español (Colectivo Urdimbre, 2000; Chamorro y otros, 2003; Moss y otros, 2003; Vallejos Llobet, 2004). En estos estudios también se han señalado las dificultades que entraña la interpretación de las nominalizaciones metafóricas $^{2}$ para lectores neófitos del discurso científico y escolar. Esto se debe a que este tipo de metáfora gramatical alude a un proceso o cualidad mediante un sustantivo en vez de la realización congruente, que sería, en caso del proceso, un verbo y, en caso de la cualidad, un adjetivo. Estas dificultades se hacen evidentes en las interacciones texto-maestro-estudiante que han sido objeto de análisis en las investigaciones de nuestro grupo (Colectivo Urdimbre, 2000; Mizuno y Moss, 2009; Chamorro, Barletta y Mizuno, 2013). En la nominalización metafórica, la información se encuentra empacada, de manera que la información que congruentemente se expresaría en una cláusula con sus componentes se expresa en forma resumida en un grupo nominal. Por ejemplo, en el caso de un proceso, en la cláusula congruente, este iría acompañado de unos participantes (actores, metas, beneficiarios, etc.) y de unas circunstancias en las cuales ocurre el proceso. En la versión nominalizada, se pierde la información acerca de los participantes y, normalmente, la de las circunstancias. A esto se le llama empaque de la información. Al proceso inverso (convertir la nominalización en una forma congruente) se le denomina desempaque (Chamorro y otros, 2003; Halliday, 2004a, 2004b).

Consideremos, primero, un ejemplo de nominalización metafórica de procesos

[1] Las difíciles condiciones sociales creadas por

la industrialización, llevaron a algunos intelectuales y obreros a criticar el nuevo modelo de organización económica y social ( H. 23.1 $)^{3}$.

Para comprender este pasaje, un lector inexperto debe realizar desempaques sucesivos así como se va abriendo la muñeca rusa hasta tener a la vista la serie completa de muñecas:

La industrialización creó difíciles condiciones sociales.

Las industrias aumentaron y esto creó difíciles condiciones sociales.

Intelectuales y obreros criticaron el nuevo modelo de organización económica y social.

Intelectuales y obreros criticaron el nuevo modelo para organizar la economía y la sociedad.

2 Especificamos nominalizaciones metafóricas para distinguirlas de aquellas nominalizaciones que se consideran congruentes (Martin, 2008; Halliday y Matthiessen, 1999).

3 Los números que se dan entre paréntesis al final de los ejemplos, se refieren a la página y el párrafo donde aparece la cita y la letra indica el texto: H. 23.1 indica página 23, párrafo 1 del texto Hipertexto. En los ejemplos de nominalizaciones, resaltamos en negrilla el grupo nominal que contiene la nominalización y subrayamos el sustantivo que sirve de Cosa del grupo nominal. 
En el ejemplo a continuación, [1a], se presenta una posible forma congruente:

[1a] Las industrias aumentaron y esto creó difíciles condiciones sociales, por lo que algunos intelectuales y obreros comenzaron a criticar el nuevo modelo para organizar la economía y la sociedad.

No obstante los desempaques realizados, aún quedan vacíos de información. ¿En qué consisten las difíciles condiciones sociales? ¿Las industrias aumentaron por sí solas? ¿Cuál es el nuevo modelo de organizar la economía y la sociedad?

Si bien es cierto que el "modelo de organización”, el capitalismo, ya se ha mencionado previamente (en la página 11, es decir, 12 páginas antes), para que el estudiante pueda procesar esta información sería necesario que el texto la reiterara o, en su defecto, que el maestro realice la mediación adecuada para complementar la información.

Ahora, revisemos la nominalización de cualidades. En la siguiente oración, en la que tenemos un ejemplo de esto (impopular $\rightarrow$ impopularidad), podemos apreciar cómo se constituye en otra dificultad para el lector inexperto.

[2] La impopularidad y las represalias de los Borbones provocaron el regreso de Napoleón de la isla de Elba, donde estaba desterrado (C. 19.1).

El problema es lo que podríamos llamar direccionalidad. Aquí encontramos dos sustantivos asociados con los Borbones, "impopularidad" y "represalias". El lector poco experto debe desempacar el sentido de la información en varios pasos:

- Debe inferir que la nominalización “impopularidad" alude a un sentimiento de la población dirigido hacia los Borbones: Ios Borbones no eran apreciados por el pueblo = los Borbones se volvieron impopulares.

- Debe inferir que el segundo sustantivo "represalias" hace referencia a las acciones di- rigidas por los Borbones hacia la población: Ios Borbones tomaron represalias contra el pueblo.

- Debe realizar el desempaque completo:

[2a] Los Borbones se volvieron impopulares, debido a las represalias que tomaban contra el pueblo, lo cual hizo que Napoleón regresara de la isla de Elba, en donde estaba desterrado.

Como podemos apreciar, en esta oración la preposición "de" representa a la vez dos relaciones lógicas diferentes, las cuales deben ser inferidas por el lector. Inferencia que puede resultar fácil de realizar para el lector experto o conocedor de la temática; no así para el novato (Billig, 2013).

El problema de la ambigüedad de la preposición "de" se asocia frecuentemente con la nominalización, debido a que las personas u objetos que, en un texto congruente, aparecen como participantes se presentan en el texto nominalizado en forma de modificador, generalmente unido a la nominalización por "de". Este fenómeno lo describimos en Moss y otros (2003) de la siguiente manera:

[La preposición 'de'] puede expresar diferentes tipos de relación. En los ejemplos que siguen, el participante (Napoleón) tiene una relación diferente con cada uno de los procesos nominalizados, pero todas estas relaciones son expresadas por medio de la misma preposición 'de'; por consiguiente, el lector debe inferir cuál es la relación que existe en cada caso:

- la llegada de Napoleón (Napoleón es sujeto del proceso; = Napoleón Ilegó)

- la derrota de Napoleón (Napoleón es complemento; = alguien (no se sabe quién) derrotó a Napoleón)

- el estudio de Napoleón ('acerca de'; = ustedes, los estudiantes, estudian la vida de Napoleón)

- el Imperio de Napoleón (poseedor; = Napoleón tenía un Imperio)

Para el lector versado en la materia, resulta evidente la relación en cada caso; no así para el lector neófito (2003: 88-89). 


\subsection{Verbos causales}

El segundo tipo de metáfora gramatical ideacional lo constituye el uso de los verbos causales (metáfora lógica, Martin, 1992; Martin y Rose, 2003). De acuerdo con Halliday (1994, 2004b), la expresión de relaciones de causa-efecto por medio de verbos causales constituye una metáfora gramatical, ya que la forma congruente de este tipo de relaciones consiste en enunciar cada uno de los hechos relacionados por medio de su correspondiente proceso (material, mental, verbal, conductual, existencial) y establecer la relación de causalidad con el uso de una conjunción adecuada. Uno de los efectos del uso del verbo causal es que, debido a la concomitante nominalización de los dos procesos involucrados, se presenta la relación como un hecho acabado, no susceptible de cuestionamientos ni de interpretaciones diversas. Esta configuración de nominalizaciones relacionadas por un verbo causal es típica del lenguaje de la ciencia y llega a constituir lo que Halliday (2004a: 111, 112) denomina síndrome o cláusula favorita de la ciencia. Veamos un ejemplo.

\section{FIGURA 1}

\section{Ejemplo [3]}

\begin{tabular}{c|l|l}
$\begin{array}{c}\text { La aparición de } \\
\text { las máquinas }\end{array}$ & significó & $\begin{array}{l}\text { una gran ruptura } \\
\text { con las tradicionales } \\
\text { formas de produc- } \\
\text { ción }(\mathrm{H} .15 .1)\end{array}$ \\
\hline Causa & & Consecuencia \\
\hline Nominalización & Verbocausal & Nominalización
\end{tabular}

\section{FIGURA 2}

Ejemplo [3а]

\begin{tabular}{c|c|l}
$\begin{array}{c}\text { Aparecieron } \\
\text { las máquinas }\end{array}$ & $\begin{array}{c}\text { y por } \\
\text { consiguiente }\end{array}$ & $\begin{array}{l}\text { se rompieron las tra- } \\
\text { dicionales formas de } \\
\text { producir (cosas) }\end{array}$ \\
\hline Causa & & Consecuencia \\
\hline $\begin{array}{c}\text { Proceso } \\
\text { material }\end{array}$ & $\begin{array}{c}\text { Conjunción } \\
\text { casual }\end{array}$ & Proceso material
\end{tabular}

La figura I muestra la forma metafórica de la oración tal como aparece en el texto mientras que la figura 2 muestra la forma congruente, desempacada, de la misma información. La forma congruente hace evidente la necesidad de desempacar los dos elementos relacionados a través del verbo causal en la forma metafórica ("aparición de las máquinas" y "ruptura con las tradicionales formas de producción”). Al utilizar la correspondiente conjunción, lo que se relacionan son dos cláusulas que incluyen sus respectivos procesos: "aparecieron" y "se rompieron". Incluso, la forma congruente todavía oculta información no suministrada por el texto; por ejemplo, el uso del clítico se en la cláusula "se rompieron las tradicionales formas de producir (cosas)" da la apariencia de que este proceso se realizó por sí solo; sin embargo, la experiencia nos indica la presencia de personas involucradas en él. Tampoco se informa qué se producía.

En el ejemplo [4], encontramos un proceso compuesto, "permitió agilizar", en el que ambos componentes son de tipo causal.

[4] La desmotadora de algodón permitió agilizar el proceso de separar la semilla de la fibra (M. 38.2).

Al desempacar los verbos causales, nos encontramos con el hecho de que la oración sigue siendo metafórica ya que aparecen dos nominalizaciones y un proceso sin participantes ("se hizo"):

[4a] Se hizo más rápido el proceso de separar la semilla de la fibra del algodón debido al uso de la desmotadora.

Para lograr una versión realmente congruente de la proposición, se hace necesario introducir el agente humano que estaba enmascarado en la versión metafórica, así:

[4b] Los industriales lograron separar la semilla de la fibra del algodón más rápidamente porque empezaron a utilizar la desmotadora.

De esta manera, podemos apreciar cómo el texto encierra diversos niveles de metaforicidad, los cuales sirven para despersonalizar los proce- 
sos omitiendo toda referencia a los actores humanos involucrados.

\section{Nuestro análisis de la metáfora gramatical}

\subsection{La nominalización}

Como describimos arriba en la sección 2, la nominalización metafórica consiste en la expresión, por medio de sustantivos, de significados que tendrían su expresión congruente en forma de verbos (procesos) o adjetivos (cualidades). En el ejemplo que sigue, vemos que el autor, en vez de utilizar el verbo "transformar", escoge la nominalización "transformaciones", convirtiendo así el proceso en una entidad que sirve como cosa o núcleo del grupo nominal "transformaciones económicas, sociales y culturales"; además, gracias a esta conversión en entidad, puede funcionar como Actor del proceso "asomar".

[5] En el siglo XVIII, la sociedad británica experimentó una serie de transformaciones económicas, sociales y culturales que asomaban por todos lados (...) (H. 11.1)

En nuestros análisis, hemos considerado pertinente diferenciar entre nominalizaciones simples y complejas; esta clasificación se basa en el criterio de la complejidad del proceso de desempaque necesario para lograr la comprensión de la nominalización. Inicialmente, definimos como nominalización compleja "la que se encuentra modificada por una cláusula o grupo cualificador de más de tres palabras (...); la que incluye dos o más nominalizaciones en un solo constituyente de cláusula (...), y las que combinan ambas características" (Moss y otros, 2003: 90). En elaboraciones posteriores, hemos especificado que "más de tres palabras" en el primer caso definido se refiere a tres o más ítems léxicos, ya que los ítems gramaticales generalmente no dificultan el proceso de interpretación del significado4. Los ejemplos [6] y [7] abajo son de nominalizaciones simples mientras que los ejemplos [8], [9] y [10] muestran nominalizaciones complejas.
[6] Esta corriente centró su atención en los efectos de la Revolución Industrial sobre los trabajadores (H. 23.2)

[7] La Revolución Industrial fue un proceso que tuvo su origen en Gran Bretaña a mediados del siglo XVIII (...) (H. 12.2).

[8] Su posición geográfica, así como el desarrollo de una gran flota mercantil y una poderosa Armada Real que se impuso a otros países europeos, le permitió a Gran Bretaña dominar los mares y las rutas comerciales marítimas más importantes (H. 12.6)

[9] En esta fase se desarrollaron las llamadas industrias pesadas: carbón, hierro y acero. Estas industrias permitieron la formación, la consolidación, el desarrollo y la difusión de la industrialización (H. 17.1).

[10] Este sistema se extendió y significó una ruptura con las formas tradicionales de vida: creciente urbanización, gran diversidad de artículos, cambios culturales y de patrones de comportamiento relacionados con el trabajo y las nuevas necesidades de consumo creadas por el capitalismo (H. 24.2).

Con respecto a las nominalizaciones complejas, podemos observar que, en el caso del ejemplo [8], la Cosa o núcleo del grupo nominal está modificada por una expresión que contiene 10 ítems léxicos. En el ejemplo [9] el grupo nominal está formado por 5 nominalizaciones y, en el ejemplo [10], tenemos un grupo nominal que contiene 5 nominalizaciones además de 6 ítems léxicos no nominalizados. Hemos considerado pertinente diferenciar entre nominalizaciones simples y complejas debido a la dificultad que involucran las últimas para el proceso de desempaque, necesario para su comprensión por parte del lector. Si tomamos el ejemplo [8], podemos ver que el procesamiento de la cantidad de información contenida en el grupo nominal hace difícil la comprensión de la oración, sobre todo para el caso de lectores inexpertos tanto en lectura como en la temática bajo estudio. Las difi- 
cultades inherentes en la interpretación de este grupo nominal incluyen las siguientes:

- El alto número de ítems léxicos resulta exigente en términos de memoria de trabajo.

- El grupo nominal incluye una cláusula hipotáctica, lo que hace más compleja su interpretación

- Es necesario desempacar la nominalización "desarrollo" e identificar su Actor.

- Es necesario saber interpretar el uso metafórico del verbo "permitir" (ver sección 2.2 arriba).

La versión congruente de esta oración se dividiría en dos o tres oraciones (ejemplo [8a]), haciendo explícitas la agencialidad del proceso "desarrollar" y la relación de causa y consecuencia. Por lo tanto, sería más fácilmente procesable.

[8a] Gran Bretaña desarrolló una gran flota mercantil y una poderosa Armada Real que se impuso a otros países europeos. Además, tenía una posición geográfica ventajosa. Por estos motivos, pudo dominar los mares y las rutas comerciales marítimas más importantes.

En el caso del ejemplo [9], se trata de desempacar las 5 nominalizaciones, tarea nada fácil ya que el texto no proporciona información acerca de los participantes de los procesos nominalizados. La primera oración aclara cuáles son las industrias mencionadas pero, como su proceso está expresado de manera no ergativa (sin explicitar la identidad del agente responsable, Halliday, 2004b: 288), tampoco da indicios de quiénes son los participantes. Además, parece existir cierta circularidad al decir que las industrias permitieron la industrialización. Como en el caso anterior, también es necesario desempacar el uso metafórico del verbo "permitir". Una posible versión congruente se presenta como ejemplo [9a]

[9a] Un grupo de personas desarrolló las industrias pesadas del carbón, el hierro y el acero. Gra- cias a estas industrias, otras personas pudieron formar, consolidar, desarrollar y difundir otras industrias.

La vaguedad de la versión congruente con respecto a la identidad de los participantes resalta aun más la falta de información en la versión nominalizada. La concentración de nominalizaciones en esta oración, entonces, dificulta enormemente la interpretación y comprensión de la información por parte de los estudiantes.

En cuanto al ejemplo [10], encontramos la combinación de los dos fenómenos ejemplificados en [8] y [9]: una concentración de nominalizaciones y, además, una serie de otros ítems léxicos que las modifican, con el resultado de que se presenta una gran densidad de información. Por otra parte, en la oración o complejo de cláusulas, esta nominalización compleja viene precedida por tres nominalizaciones simples: "ruptura", "creciente urbanización" y "gran diversidad de artículos"; una cláusula no ergativa (ver Cláusulas no ergativas, sección 3.3), y un verbo causal. El proceso de desempaque, entonces, implica desempacar varias nominalizaciones, tener en cuenta los significados de todos los ítems léxicos, desempacar el verbo causal e inferir el agente de la cláusula no ergativa. De esta manera, resulta un proceso de interpretación muy exigente para el lector. Una posible versión congruente se presenta en el ejemplo [10a].

[10a] La sociedad británica fue adoptando el nuevo sistema económico capitalista y por esta razón rompió con las formas tradicionales de vida. Los centros urbanos crecieron, las fábricas producían artículos muy diversos, la cultura cambió y también la forma como la gente se comportaba. Todo esto tenía que ver con el trabajo y las cosas que la gente sentía que necesitaba consumir como resultado del capitalismo.

Como se puede apreciar, se necesitan tres oraciones para expresar estos significados de manera congruente. Por supuesto, no estamos sugiriendo que un lector experto realice este tipo de transformaciones al momento de leer, 
pero la versión congruente es un indicio de la complejidad y densidad de la información contenida en este tipo de metáfora gramatical y de la dificultad que implica su interpretación para lectores no expertos. Por este motivo consideramos importante, al momento del análisis, la diferenciación entre nominalizaciones simples, relativamente fáciles de desempacar, y nominalizaciones complejas. Las nominalizaciones complejas forman parte de un síndrome de metáforas gramaticales presentes en estos textos escolares que, sugeriremos, llegan a conformar una conspiración gramatical (Martin, 1988; Moss, 2010). Dicha conspiración excluye a los estudiantes del acceso a la información acerca de participantes responsables y circunstancias que permitiría la comprensión adecuada de los eventos históricos descritos.

\subsection{Los verbos causales}

Siguiendo a Halliday (1994, 2003a, 2004b), consideramos como metáfora gramatical la expresión de la causalidad por medio de verbos en vez de conjunciones de causa-efecto. Este tipo de metáfora es muy frecuente en los textos bajo estudio. Algunos ejemplos se encuentran en [11], [12] y [13].

[11] La aparición de las máquinas significó una gran ruptura con las tradicionales formas de producción (H. 15.1).

[12] La construcción de ferrocarriles, a partir de 1830, estimuló el desarrollo de las industrias textiles y siderúrgicas (H. 19.5)

[13] Las transformaciones técnicas en la agricultura, y la utilización de mejores abonos, originan una alta productividad agrícola y desarrollan una agricultura de exportación, la cual viene a satisfacer el mercado de alimentos, interno y externo, de Inglaterra (M. 37.5).

El proceso de desempaque de estas metáforas de causalidad implica también el desempaque de las nominalizaciones que constituyen la mayoría de los participantes asociados con los procesos. Esta combinación de metáforas lógicas (verbos causales) y metáforas experienciales (nominalizaciones) se caracteriza por un alto grado de abstracción (Martin, 1992: 407). Por ejemplo, [11] se podría desempacar de la siguiente manera:

[11a] Las máquinas fueron inventadas y, como consecuencia, dejaron de usarse las tradicionales formas de producir las cosas.

Sin embargo, el proceso significó lleva consigo una ambigüedad entre los sentidos de simbolizar/equivaler y causar. El desempaque [11a] representa el sentido de causar; por la naturaleza semiótica de la relación de simbolizar, resulta difícil, si no imposible, desempacar la oración con este sentido.

En este caso, podemos apreciar que el desempaque hace más evidente tanto la ambigüedad del proceso como el hecho de que el texto no especifica los participantes humanos de los procesos. No se nos informa ni quién inventó las máquinas ni quiénes dejaron de usar las formas tradicionales de producción. [13].

Algo parecido ocurre con los ejemplos [12] y

[12a] A partir de 1830 fueron construidos muchos ferrocarriles; como resultado de esto, las industrias textiles y siderúrgicas pudieron desarrollarse.

[13a] Las técnicas utilizadas en la agricultura fueron transformadas y empezaron a utilizarse mejores abonos. Por esta razón, la agricultura se volvió más productiva y, por lo tanto, fue posible exportar productos agrícolas y así satisfacer el mercado de alimentos, interno y externo, de Inglaterra.

En estos dos ejemplos, de igual manera, el desempaque demuestra claramente la ausencia de participantes humanos en la mayoría de los casos, creando lo que Billig (2013) denomina "texto despoblado"; además, en el caso del ejemplo [13], la versión desempacada [13a] aclara la secuencia temporal de los eventos, secuencia poco clara en la versión original. Ahora bien, parece 
probable que este tipo de metáfora gramatical represente un menor nivel de dificultad para la comprensión que las nominalizaciones complejas. Sin embargo, el proceso de desempaque realza ciertas características interesantes de la información contenida en los textos, u omitida en ellos.

\subsection{Cláusulas no ergativas}

En nuestros análisis de la metáfora gramatical, hemos incluido algunas categorías que no menciona Halliday explícitamente (Moss, 2010); una de ellas es la de las cláusulas no ergativas. Como parte del análisis de la transitividad, diferenciamos entre cláusulas ergativas, que hacen explícita la agencialidad, y cláusulas no ergativas, que dejan implícita dicha agencialidad o sugieren que no existe agente externo con relación al proceso. Hasta ahí seguimos a Halliday (1994, 2004b) y a Thompson (2004). Nosotros consideramos, además, que las cláusulas no ergativas son metafóricas en aquellos casos en que nuestros conocimientos nos indican que debe existir un agente externo cuya presencia es ocultada por las escogencias lexicogramaticales del autor. Veamos unos ejemplos:

[14] La riqueza aumentaba y se acumulaba mientras miles de hombres, mujeres y niños trabajaban desesperanzados y hambrientos (H. 11.1).

En este ejemplo, los procesos "aumentaba" y "se acumulaba" se relacionan con un solo participante: la riqueza. Es decir, el texto parece sugerir que la riqueza aumentaba y se acumulaba por sí sola. Sin embargo, nuestros conocimientos nos indican que, en el mundo real, esto no es así; la riqueza no tiene ni voluntad ni capacidad de actuación sino que son ciertas personas las que la aumentan y acumulan. En este caso, entonces, existe en el mundo real un participante - ciertas personas - que no aparece en el texto. Thompson (2003) propone diferenciar entre "W-participants" (World-participants) y "Cparticipants" (Clause-participants) y sugiere que, en casos donde existen W-participants que no aparecen como C-participants, se trata de metá- fora gramatical. Nuestra propuesta con respecto a las cláusulas no ergativas es parecida. Veamos otros ejemplos.

[15] Con la Ley de Cercamientos de 1727, se legalizaron estas apropiaciones (H. 13.2).

[16] Por ejemplo, en 1700 se prohibió la entrada de textiles de la India, y en 1813 se obligó a esta colonia a importar masivamente tejidos de algodón del Lancashire (H. 14.4).

En ambos ejemplos se trata de medidas tomadas por el Parlamento británico, es decir, por un grupo de hombres representantes de la burguesía y la aristocracia británicas; sin embargo, estos participantes no aparecen en el texto. Solamente aparecen las medidas sin información con respecto a quién las tomó. Ahora bien, un lector experto con conocimientos de la historia británica puede inferir fácilmente la información faltante. No así lectores inexpertos sin conocimientos previos, para quienes resultaría más informativa la versión congruente que se sugiere a continuación:

[15a] Con la Ley de Cercamientos de 1727, el Parlamento británico legalizó estas apropiaciones.

[16a] Por ejemplo, en 1700, el Parlamento británico promulgó una ley que prohibió la entrada de textiles de la India y en 1813 adoptó medidas para obligar a esta colonia a importar masivamente tejidos de algodón del Lancashire.

La incongruencia gramatical presente en estos tipos de cláusula no ergativa y que nos lleva a considerarlos como casos de metáfora gramatical se muestra en forma gráfica en la figura 3, abajo.

\subsection{Presente histórico}

En los textos de historia, es de esperar que la mayor parte de los procesos aparezcan en tiempo pasado. Sin embargo, en algunos de los textos que hemos analizado, hemos encontrado un uso frecuente del presente simple en sentido histórico. Consideramos que este uso se constituye en un caso de metáfora gramatical por la incongruencia que se produce entre el tiempo 
gramatical utilizado y la referencia temporal del contenido del texto, creando así la tensión entre los estratos semántico y lexicogramatical que caracteriza a la metáfora gramatical (ver figura 3, abajo).

Observemos el siguiente párrafo:

[17] El desarrollo de la técnica genera una serie de repercusiones sociales y económicas. En el orden social se producen cambios radicales, debido al surgimiento de la clase obrera, formada en el trabajo de la industria textil; también se fortalece y evoluciona la clase de los mercaderes en una nueva: la burguesía, propietaria de los medios de producción (tierra, maquinaria y capital) y con capacidad de compra de mano de obra a través del salario (M. 38.7)

El párrafo forma parte de una sección que se intitula "Fases de la Revolución Industrial" y se refiere a las transformaciones sociales y económicas que ocurrieron en Gran Bretaña durante la Revolución Industrial. Sin embargo, el uso sostenido del presente histórico puede prestarse para confusiones a la hora de interpretar, dando la impresión de que se están presentando reglas universales de la ciencia social.

\subsection{Participantes no animados}

Ciertos tipos de proceso, sobre todo los procesos mentales y, en algunos casos, los procesos conductuales y verbales, lógicamente sugieren la presencia de un participante humano o, por lo menos, animado. Cuando dichos procesos aparecen en los textos asociados con participantes no animados, hemos considerado que se trata de una clase de metáfora gramatical, debido a la incongruencia entre la clase de participantes y la clase de proceso (ver figura 3, abajo). A continuación, presentamos algunos ejemplos:

[18] La Revolución Industrial, que originó el sistema económico capitalista industrial, nació en Inglaterra a finales del siglo XVIII, entre $1780 \mathrm{y}$ 1790 (M. 37.2).

[19] La productividad exigía cada vez más la búsqueda de nuevos mercados y materias primas ( $M$. 39.3).
[20] La industria del hierro disfrutó una serie de mejoras técnicas (H. 17.4).

[21] La industria algodonera se vio estimulada y perfeccionada con el invento de la máquina desmotadora, del estadounidense Whitney (H. 19.6).

Podría argumentarse que, en estos casos, se trata simplemente de metáforas léxicas o metonimias y no metáforas gramaticales. Sin embargo, llama la atención que, para desempacar estas metáforas y elaborar una versión congruente de estas oraciones, es necesario utilizar otro tipo de proceso y/o introducir los participantes humanos implícitos en la versión metafórica, como se puede apreciar a continuación:

[18a] La Revolución Industrial, que originó el sistema económico capitalista, tuvo su origen en Inglaterra a finales del siglo XVIII, entre 1780 y 1790.

[19a] El hecho de que las fábricas produjeran más hacía cada vez más necesario que los dueños de las industrias buscaran nuevos mercados y materias primas.

[20a] Los dueños de la industria del hierro aplicaron una serie de mejoras técnicas

[21a] Los dueños de la industria algodonera recibieron un estímulo y pudieron perfeccionar sus procesos con el invento de la máquina desmotadora, del estadounidense Whitney.

En los casos de [18] y [19], el proceso de desempaque implica cambiar de procesos conductuales [18] o verbales [19] a procesos relacionales. En los casos de [19], [20] y [21], se hace necesario explicitar la referencia a los dueños de las industrias, es decir, a los participantes animados. Estas diferencias gramaticales entre las versiones metafóricas y congruentes nos hacen clasificar estos casos como metáforas gramaticales.

\section{4. ¿Por qué este tipo de análisis?}

Nuestros objetivos al realizar este tipo de análisis de la metáfora gramatical en los textos escolares de Ciencias Sociales son tres:

- Profundizar en la comprensión del fun- 


\section{FIGURA 3}

Incongruencia de expresiones no ergativas, presente histórico y participantes no animados 5

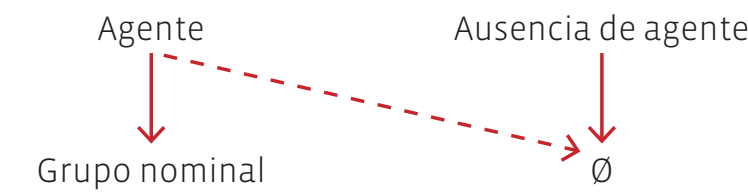

Proceso en pasado Proceso en presente

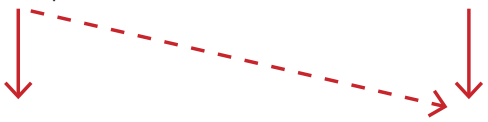

Tiempo pasado

Tiempo presente

Actuante/Emisor

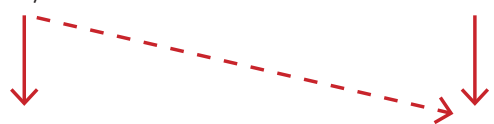

Sustantivo animado

Sustantivo inanimado

\begin{tabular}{|c|l|}
\hline$\longrightarrow$ & Realización congruente \\
\hline$-------\rightarrow$ & Realización incogruente \\
\hline
\end{tabular}

cionamiento de la metáfora gramatical en lengua española.

- Analizar las implicaciones del uso de la metáfora gramatical en términos de la ideología de la historia y de la ciencia que subyace en el texto.

- Analizar las implicaciones del uso de la metáfora gramatical para los procesos de comprensión y aprendizaje a partir del texto de parte de los alumnos a quienes va dirigido.

\subsection{La metáfora gramatical en lengua española}

Por medio de estos análisis hemos podido definir 5 clases de metáfora gramatical en español: la nominalización, los verbos causales, las cláusulas no ergativas, el presente histórico y los participantes no animados. Las dos primeras han sido ampliamente reconocidas en la litera- tura de la Lingüística Sistémico-Funcional tanto en inglés (Eggins y otros, 1993; Halliday y Martin, 1993; Martin, 2003; Halliday, 2004b) como en español (Colectivo Urdimbre, 2000; Chamorro y otros, 2003; Moss y otros, 2003; Vallejos Llobet, 2004). La tercera, las cláusulas no ergativas, ha sido planteada para lengua inglesa por Thompson (2003). Nuestra propuesta, entonces, consiste en aplicar a la lengua española la teoría de Thompson con respecto a la metaforicidad de cláusulas que tienen $W$-participants implícitos que no aparecen como C-participants, e incluir en la categoría de metáfora gramatical en español el uso sostenido del presente histórico y los participantes no animados con procesos que típicamente exigen participantes animados.

En este sentido, consideramos que la práctica de elaborar versiones congruentes de cláusulas metafóricas es una ayuda importante en el proceso de identificar la metáfora gramatical.

\subsection{Implicaciones ideológico-educativas de la metáfora gramatical}

En varias publicaciones (Chamorro y otros, 2003; Moss y otros, 2003; Moss, 2010) hemos abordado la cuestión de las implicaciones ideológicas del uso de la metáfora gramatical en los textos escolares de Ciencias Sociales. Moss (2010) sugiere la existencia de una "conspiración gramatical" (cf. Martin, 1988) en la que la co-ocurrencia de las cinco clases de metáfora gramatical identificadas arriba, junto con otras características de estos textos, promueve una visión determinista de la historia que presenta los eventos como hechos de la naturaleza, determinados por leyes externas sin intervención humana (Gardiner, 1959). En este orden de ideas, hemos visto que típicamente:

- la nominalización metafórica evita identificar los actores humanos responsables de los hechos;

- la nominalización metafórica presenta 
las acciones y sucesos no como procesos, sino como hechos acabados, como objetos;

- los verbos causales tal vez no tengan en sí mismos implicaciones ideológicas pero conllevan a la nominalización de los dos eventos relacionados como causa y consecuencia;

- Ias cláusulas no ergativas sugieren que los hechos ocurren por sí solos, por generación espontánea y no dejan ver los intereses humanos involucrados, ni de Actores ni de Afectados;

- el presente histórico, cuando se utiliza de manera sostenida, da la impresión de referirse a leyes universales y no a eventos particulares;

- el uso de participantes no animados con procesos mentales o conductuales sugiere que dichos participantes poseen voluntad propia.

Si la misión de la educación es la formación de ciudadanos con capacidad para participar activamente en la construcción de la sociedad, desarrollar su identidad, defender sus derechos, acceder y contribuir a los bienes y servicios de la comunidad, se esperaría que los textos escolares representaran a los seres humanos involucrados en procesos de crear, transformar, pensar, decir, actuar, que construyan al ser humano como agente promotor, instigador y ejecutor de cambios en la sociedad. Es decir, sería más apropiada una visión de la historia como construida por los seres humanos y en la que la agencialidad humana se someta a investigación, análisis y crítica (Said, 2003: xxii).

Por otra parte, mediante la escogencia de estas formas metafóricas, los autores esconden o enmascaran tanto la participación humana en los procesos sociohistóricos como también la participación de investigadores historiadores en la interpretación de dichos procesos. Presentan resultados de la investigación histórica como si fuesen simplemente hechos de la naturaleza sin reconocer que se trata de una interpretación entre varias posibles de la realidad del pasado. Pensamos que sería más productivo, en términos de la formación de posibles futuros investigadores, que se hiciera visible la actividad científica que subyace a la información presentada en el texto y se discutieran diversas opciones para validar este tipo de información (cf. Coffin, 2006).

\subsection{Implicaciones de la metáfora gramatical para la comprensión y el aprendizaje}

Las dificultades que pueden presentarse para la comprensión de los textos metafóricos por parte de alumnos no expertos, ni en lectura ni en la temática de los textos, se han documentado ampliamente en la literatura, tanto en inglés como en español. Aquí resumimos algunas de ellas y comentamos acerca de sus implicaciones para la práctica del docente en el aula:

- Se hace necesario que el alumno aprenda a desempacar las metáforas para acceder al significado del texto. En nuestra experiencia investigativa en 11 contextos de aula, hemos observado que esto implica un proceso largo de "entrenamiento" por parte de un profesor consciente de las dificultades y dispuesto a dedicarle tiempo a este aspecto del aprendizaje. Además, en los 10 textos que hemos analizado, frecuentemente la información necesaria para realizar el desempaque de las metáforas no aparece en ninguna parte. El docente, entonces, debe proveer la información faltante o guiar a los alumnos para buscarla en otras fuentes.

- Para ir desarrollando una consciencia crítica, es importante que los estudiantes se acostumbren a formularse preguntas con respecto a la información que no está explícita en el texto. El profesor debe indagar constantemente acerca de los participantes humanos de los eventos relacionados en el texto, sus intereses, roles y motivaciones. También es importante que promueva la reflexión acerca de los efectos de los hechos descritos y de otras posibles interpretaciones de los mismos.

\section{Conclusión}

La metáfora gramatical es una poderosa herramienta en la elaboración del discurso cien- 
tífico, ya que ayuda a establecer relaciones entre fenómenos de manera más fácil, así como construir sistemas de clasificación de forma más adecuada. Sin embargo, se puede convertir en un factor de dificultad para el aprendizaje de las ciencias en la escuela. Según Halliday (1998), una de las principales barreras que se le presentan al estudiante de la escuela secundaria para acceder al conocimiento técnico y disciplinar es aprender a manejar la gramática nominalizada del discurso científico. Así, el discurso altamente metafórico y abstracto de los textos analizados limita el acceso al conocimiento para gran parte de los estudiantes que no están familiarizados con estas formas de decir (Martin, 1992; Halliday, 2003b; Fairclough, 2008). Ahora bien, la identificación de recursos metafóricos no permite por sí sola entender la estructura del conocimiento disciplinar; pero sí constituye un paso inicial para que los estudiantes puedan comprender los contenidos de los textos, comprensión indispensable para acceder a la forma como se construye el conocimiento en el área del saber. Asimismo, la falta de acceso al discurso metafórico también limita el acceso al poder asociado con su uso. Por ello, proponemos un manejo apropiado de este recurso lingüístico en sus distintas formas de expresión (nominalización, verbos causales, presente histórico, participantes no animados, expresiones no ergativas), especialmente en lo que tiene que ver con su desempaque, un proceso que evidencia la información que oculta el proceso de metaforización. Este manejo puede contribuir a la formación de ciudadanos críticos que tengan la capacidad de reflexionar sobre lo que leen y saber que existen otras interpretaciones de los hechos.

\section{Bibliografía citada}

BILLIG, Michael, 2008: "The language of critical discourse analysis: the case of nominalization", Discourse \& Society 19 (6), 783-800.

BILLIG, Michael, 2011: "Writing social psychology: Fictional things and unpopulated texts", British Journal of Social Psychology 50, 4-20.

BILLIG, Michael, 2013: Learn to write badly. How to succeed in the social sciences, Cambridge: Cambridge University Press.

Chamorro, Diana, Jorge Mizuno y Gillian Moss, 2003: "Tergiversaciones y correspondencias: la metáfora y sus bemoles", Revista Latinoamericana de Estudios del Discurso 3 (1), 29-47.

Chamorro, Diana, Norma Barletta y Jorge Mizuno, 2013: "El lenguaje para enseñar y aprender las Ciencias Naturales: un caso de oportunidades perdidas para la formación ciudadana", Revista Signos 46 (81), 3-28.

Coffin, Caroline, 2006: Historical Discourse. The language of time, cause and evaluation, London: Continuum.

Colectivo URdimbre, 2000: Libros de texto y aprendizaje en la escuela, Sevilla: Díada Editores.

Colombl, Cecilia, 2000: El desarrollo del registro académico del español en estudiantes latinos en EE.UU. Ponencia presentada en el II Congreso Internacional de la Lengua Española, Valladolid, España [http://cvc.cervantes.es/obref/ congresos/valladolid/ponencias/unidad_diversidad_del_espanol/3_el_espanol_en_los_EEUU/ colombi_m.htm, fecha de consulta: 8 de agosto de 2012].

Eggins, Suzanne, Peter Wignell y Jim R. Martin, 1993: "The discourse of history: distancing the recoverable past" en Mohen Ghadessy (ed.): Register analysis: Theory and practice, London: Pinter, 75109.

FaIRclough, Norman, 2008: "The language of critical discourse analysis: reply to Michael Billig", Discourse \& Society 19 (6), 811-819.

Gardiner, Patrick (ed.), 1959: Theories of History, Glencoe, Illinois: The Free Press.

Halliday, Michael A. K., 1994: An Introduction to Functional Grammar, 2nd edition, London: Arnold.

Halliday, Michael A. K., 1998: "Things and rela- 
tions: regrammaticising experience as technical knowledge" en Jim R. Martin y Robert Veel (eds.): Reading science. Critical and functional perspectives on discourses of science, London: Routledge, 185-235.

Halliday, Michael A. K., 2003a: "On the 'architecture' of human language” en Jonathan WeBSTER (ed.): On Language and Linguistics (vol. 3 de Collected Works of M. A. K. Halliday), London and New York: Equinox, 1-29.

HallidaY, Michael A. K., 2003b: "Is the grammar neutral?" en Jonathan Webster (ed.): On Language and Linguistics (vol. 3 de Collected Works of M. A. K. Halliday), London and New York: Equinox, 271292.

Halliday, Michael A. K., 2004a: The Language of Science (vol. 4 de Collected Works of M. A. K. HaIliday), ed. Jonathan Webster. London: Continuum. Halliday, Michael A. K., 2004b: An Introduction to Functional Grammar, 3rd edition, revised by C. M. I. M. Matthiessen, London: Arnold.

Halliday, Michael A. K. y Jim R. Martin, 1993: Writing science: Literacy and discursive power, London: Falmer.

Halliday, Michael A. K. y Christian M. I. M. MatthiesSEN, 1999: Construing experience through meaning. A language-based approach to cognition, London: Continuum.

Martin, Jim R., 1988: "Grammatical conspiracies in Tagalog: Family, Face and Fate - with regard to Benjamin Lee Whorf" en James D. Benson, Michael J. Cummings y William S. Greaves (eds.): Linguistics in a systemic perspective, Amsterdam: Benjamins, 243-300.

Martin, Jim. R., 1992: English Text. System and structure, Philadelphia: John Benjamins.

MARTIN, Jim. R., 2003: "Making history: Grammar for interpretation" en Jim R. MARTIN y Ruth Wodak (eds.): Re/reading the past: Critical and functional perspectives on time and value, Amsterdam: Benjamins, 19-57.

MARTIN, Jim. R., 2008: "Incongruent and proud: devilifying 'nominalization'”, Discourse and Society 19 (6), 827-836.
Martin, Jim R. y David Rose, 2003: Working with discourse: Meaning beyond the clause, London: Continuum.

Mızuno, Jorge y Gillian Moss, 2009: “La ecología: una noticia de moda. El registro periodístico y la transitividad en un texto de ciencias naturales" en Martha Shiro, Paola Bentivoglio y Frances D. ERlich (comps.): Haciendo discurso. Homenaje a Adriana Bolivar, Caracas: Ediciones Chirimi, 647-660.

Moss, Gillian, 2010: "Textbook language, ideology and citizenship: the case of a history textbook in Colombia”, Functions of Language 17 (1), 71-93.

Moss, Gillian, Jorge Mizuno, Diana Avila, Norma Barletta, Solange Carreño, Diana Chamorro y Carlina TAPIA, 2003: Urdimbre del texto escolar: ¿Por qué resultan difíciles algunos textos?, segunda edición, Barranquilla: Uninorte.

SAID, Edward W., 2003: Orientalism, reprinted with a new preface, London: Penguin.

Thompson, Geoff, 2003: "The elided participant: Presenting an uncommonsense view of the researcher's role" en Anne-Marie Simon-VandENbergen, Miriam Taverniers y Louise Ravelli (eds.): Grammatical metaphor. Views from systemic functional linguistics, Amsterdam: John Benjamins, 257-278.

Thompson, Geoff, 2004: Introducing functional grammar, 2nd edition, London: Arnold.

Vallejos Llobet, Patricia (ed.), 2004: El discurso científico pedagógico: Aspectos de la textualización del "saber enseñado", Bahía Blanca: Universidad Nacional del Sur.

VyGotsky, Lev, 1986 [1934]: Thought and Language, Cambridge, Mass: MIT Press (original en ruso).

Wignell, Peter, 1998: "Technicality and abstraction in social science" en Jim R. Martin y Robert Veel (eds.): Reading science. Critical and functional perspectives on discourses of science, London: Routledge, 297-326. 


\subsection{Manuales escolares utilizados en la investigacion}

Civilización 8. Ciencias Sociales Integradas. 1994. Editorial Norma, Bogotá. Autores: A. Montenegro, J. Eastman, G. Mejía, M. Urrego, I. Rojas, I. Florián, J. Feo.

Hipertexto 8. Santillana. Sociales. 2010. Editorial Santillana, Bogotá. Autores: B. Caballero, J. Cote, H. Cristancho, A. Fajardo, C. Maldonado.

Milenio 8: Historia y Geografía. 1997. Editorial Norma, Bogotá. Autores: C. Ramos y O. Espinosa. 